\title{
Métodos numéricos para valoración de opciones usando esquemas débiles para Euler-Maruyama y Taylor 2.0
}

\author{
Luis E. Girón', Daniel Suescún-Díaz ${ }^{2 *}$ y Faiber Robayo-Betancourt ${ }^{3}$ \\ (1) Facultad de Ciencias Económicas y Administrativas, Departamento de Economía, Pontificia Universidad Javeriana \\ Cali, Colombia. (correo-e: legiron@javerianacali.edu.co) \\ (2) Departamento de Ciencias Naturales, Universidad Surcolombiana, Neiva, Huila, Colombia. \\ (correo-e: daniel.suescun@usco.edu.co) \\ (3) Departamento de Ingeniería Electrónica, Universidad Surcolombiana, Neiva, Huila, Colombia. \\ (correo-e: faiber.robayo@usco.edu.co)
}

* Autor a quien debe ser dirigida la correspondencia

Recibido Mar. 4, 2021; Aceptado May. 7, 2021; Versión final Jun. 20, 2021, Publicado Oct. 2021

\section{Resumen}

El objetivo en este estudio es valorar una opción de compra estándar europea en diferentes escenarios utilizando, por un lado, la fórmula desarrollada por Black-Scholes, y por el otro, los métodos numéricos estocásticos de Euler-Maruyama y Taylor 2.0 con aproximaciones débiles. Se simulan diferentes trayectorias, pasos de tiempo, tasas de interés libre de riesgo y diferentes precios de ejercicio. Los resultados obtenidos en la valoración de opciones en todos los casos analizados muestran que los métodos de Euler-Maruyama y Taylor 2.0 presentan errores cuadráticos medios muy bajos en comparación con la valoración obtenida usando la fórmula de Black-Scholes. En conclusión, los métodos de aproximación débiles son lo suficientemente precisos para ser usados en la ingeniería o en las finanzas.

Palabras clave: formula Black-Scholes; aproximación débil; método Taylor 2.0; Euler-Maruyama

\section{Numerical methods for option pricing using weak schemes for Euler-Maruyama and Taylor 2.0}

\begin{abstract}
The primary objective of this study is to evaluate a standard European call option under different scenarios by using the Black-Scholes formula and the stochastic numerical methods of Euler-Maruyama and Taylor 2.0 with weak approximations. Simulations are performed under different trajectories, time steps, risk-free interest rates, and different strike prices. The results obtained for option valuation in all the cases analyzed show that the Euler-Maruyama and Taylor 2.0 methods have very low mean square errors when compared to the valuations obtained using the Black-Scholes formula. In conclusion, weak approximation scheme methods are sufficiently precise to be used in engineering or finance.
\end{abstract}

Keywords: Black-Scholes formula; weak approximation; Taylor 2.0 method; Euler-Maruyama method 


\section{INTRODUCCIÓN}

Los métodos numéricos son una forma que permiten obtener soluciones numéricas en una variedad de aplicaciones. Dichos métodos pueden ser de naturaleza determinística o estocástica, dependiendo si la ecuación a resolver involucra o no variables aleatorias dependientes del tiempo. Dentro de los métodos numéricos determinísticos, se pueden encontrar entre otras aplicaciones: simulaciones numéricas para el comportamiento térmico (Ortega et al., 2009), simulaciones numéricas para estudiar la propagación de una fisura en el material (Palma et al., 2010), utilización del método de elementos finitos para estudiar los flujos turbulentos isotérmicos (Dos Santos et al., 2011), los métodos de diferencias finitas para resolver ecuaciones de advección-difusión no estacionarias (Bermúdez y Juárez,2014), y simulaciones numéricas para estudiar el efecto de la presión en el proceso de mezcla metano-oxígeno (De la Cruz et al., 2015). Dentro de los métodos numéricos estocásticos, las simulaciones numéricas se han aplicado en el estudio de un ferromagnético con diferentes anisotropías usando el método de Monte Carlo (De la Espriella et al., 2012), y en reactores nucleares usando el método de Euler-Maruyama (Suescún-Díaz et al., 2018).

Los métodos numéricos pueden ser usados en las opciones financieras que otorgan un derecho a su poseedor a comprar o vender un activo a un precio determinado durante un periodo o en una fecha prefijada. La opción puede ser europea si la opción se puede ejercer sólo en el vencimiento del contrato. El precio pagado por el activo cuando la opción se ejerce se denomina precio de ejercicio. El ultimo día que la opción se puede ejercer se denomina fecha de vencimiento. El principal desafío en el mercado de opciones es cómo fijar un precio justo para la opción de tal manera que se eviten oportunidades de arbitraje. Bajo una serie de supuestos (Black y Scholes, 1973), desarrollaron la fórmula para valorar opciones de compra de venta estándar. El precio de las opciones calculadas con la fórmula de (Black y Scholes, 1973), no coinciden exactamente con los calculados por el mercado, sin embargo, sigue siendo una forma de valorar hasta el momento con una buena aproximación.

Se han desarrollado diversos métodos de valoración de opciones, un método fija el precio de las opciones europeas en presencia de costos de transacción (Monoyios, 2004). Se desarrollaron métodos numéricos asumiendo que el precio del activo subyacente se comporta como un movimiento Browniano geométrico (Toivanen, 2008). Un enfoque usando los métodos explícitos en diferencias finitas (O'Sullivan y O'Sullivan, 2011). El método de diferencias finitas implícitas aplicando Crank-Nicholson en un modelo GARCH $(1,1)$ (Rana y Ahmad, 2011), el método de diferencias finitas para resolver ecuaciones integro-diferenciales parciales que describen el comportamiento de los precios de las opciones bajo modelos de difusión por salto (Kwon y Lee, 2011), el método de cambio de regímenes o estados para valoración de opciones (Zhu et al., 2012), los métodos usando la transformada de Fourier rápida (Zhang y Wang, 2013 ; Huang et al., 2014), el método martingala y la teoría de conjuntos difusos (Nowak y Romaniuk, 2014), el método que considera costos de transacción y volatilidad estocástica (Mariani et al., 2015), el método que mejora el esquema compacto de diferencias finitas de orden superior para la fijación de precios de opciones en modelos de volatilidad estocástica no afines (Shi et al., 2016), y el método que se fundamenta en el marco de equilibrio general teniendo en cuenta la volatilidad estocástica (Li et al., 2017). En este trabajo se considera la utilización del método estocástico de Taylor 2.0 (Milstein, 1985) con aproximación débil para valoración de opciones de compra estándar, tomando como punto de referencia los valores para opciones de compra arrojados por la fórmula tradicional (Black y Scholes, 1973).

\section{CONSIDERACIONES TEÓRICAS}

El modelo de (Black y Scholes, 1973), considera que el activo subyacente no paga dividendos y asume que el rendimiento continuamente compuesto de dicho activo en un periodo corto se distribuye normalmente, es decir, que su precio sigue una distribución log-normal. Adicional a los supuestos anteriores también se asume que la volatilidad durante el periodo del contrato es constante, que no hay costos de transacción ni impuestos y los activos son perfectamente divisibles. Tampoco existen posibilidades de arbitraje y los inversionistas pueden adquirir $u$ otorgar préstamos a la tasa de interés libre de riesgo $r$, la cual se supone constante. Matemáticamente, el modelo parte de la ecuación diferencial estocástica para el precio del activo subyacente (Black y Scholes, 1973),

$$
d S(t)=\mu S(t) d t+\sigma S(t) d W(t)
$$

Siendo $S(0)=S_{0}$, el valor del activo subyacente al inicio del contrato, $\mu$ es la tasa de retorno esperada, $\sigma$ es la volatilidad del retorno del activo, dW es un proceso de Wiener o movimiento Browniano. El proceso estocástico $S($.$) resuelve la ecuación (1) siempre que:$

$$
S(t)=S_{0}+\int_{0}^{t} \mu S(s) d s+\int_{0}^{t} \sigma S(s) d W s \quad ; t \geq 0
$$


Siendo la primera integral en la ecuación (2) una integral de Riemann y la segunda una integral de Itô ó integral estocástica. Para hallar la fórmula que permiten encontrar el precio teórico de una opción de compra estándar europea que no paga dividendos, se parte de la ecuación diferencial parcial de (Black y Scholes, 1973), cuya solución nos permite obtener las fórmulas buscadas:

$$
c=S_{t} N\left(d_{1}\right)-K e^{-r t} N\left(d_{2}\right)
$$

Donde,

$$
\begin{aligned}
& d_{1}=\frac{\ln \left(S_{t} / K\right)+\left(r+\frac{\sigma^{2}}{2}\right) T}{\sigma \sqrt{T}} \\
& d_{2}=\frac{\ln \left(S_{t} / K\right)+\left(r-\frac{\sigma^{2}}{2}\right) T}{\sigma \sqrt{T}}=d_{1}-\sigma \sqrt{T}
\end{aligned}
$$

Donde, $c$ es el precio de una opción de compra europea, el cual depende del valor actual del subyacente $S_{\mathrm{t}}$, el precio de ejercicio $\mathrm{K}$, el tiempo hasta el vencimiento $\mathrm{T}$, el tipo de interés libre de riesgo $\mathrm{r}$, la volatilidad de los rendimientos del subyacente $\sigma$ y de la función de distribución de probabilidad acumulada para una variable $\mathrm{x}$ con distribución normal estándar $N(\mathrm{x})$. Obsérvese, que la tasa de retorno esperada $\mu$ no aparece en las fórmulas de valoración porque se ha supuesto que los agentes son neutrales al riesgo.

\section{MÉTODOS NUMÉRICOS}

Existen diversos métodos numéricos estocásticos para aproximarnos a la solución de una ecuación diferencial estocástica, ya sea por aproximaciones fuertes o aproximaciones débiles, en este trabajo se usan las aproximaciones débiles para resolver ecuaciones diferenciales estocásticas. Una aproximación en tiempo discreto $Y^{\delta}$ converge débilmente para $X \operatorname{con}$ orden $\beta>0$, en el tiempo T cuando $\delta$ tiende a cero para cualquier función $\mathrm{g}$ de orden polinomial, sí existe una constante positiva $\mathrm{L}>0, \mathrm{~L}$ no depende del valor $\delta$ (Kloeden y Platen, 1995) tal que:

$$
\left|E\left(g\left(X_{T}\right)\right)-E\left(g\left(Y_{N}\right)\right)\right| \leq L \delta^{\beta}
$$

En el caso del método de Euler Maruyama converge con un orden débil de $\beta=1$. En finanzas se trabaja regularmente con la convergencia débil, la cual tiene la ventaja de exigir menos carga computacional, además, proporciona los momentos de orden uno y dos de la distribución, los cuales son utilizados en el cálculo de valoración de la opción (Sauer, 2011).

En este trabajo, se considera el método estocástico de Taylor 2.0 débil simplificado (Kloeden y Platen, 1995; Tocino, 2009). El método de Taylor débil de orden 2.0 se deriva de la expansión estocástica de Taylor, sin embargo, el objetivo del método no es aproximarse al proceso estocástico en su totalidad sino algunos de sus momentos. Sea un proceso de Itô autónomo $\mathrm{S}_{\mathrm{t}}$, con término de deriva $\mathrm{a}\left(\mathrm{S}_{\mathrm{t}}\right)$ y coeficiente de difusión $\mathrm{b}\left(\mathrm{S}_{\mathrm{t}}\right)$ tal como:

$$
d S_{t}=a\left(S_{t}\right) d t+b\left(S_{t}\right) d W_{t}
$$

Con condición inicial $\mathrm{S}(0)=\mathrm{S}_{0}$.

Donde,

$$
\begin{aligned}
& a\left(S_{t}\right)=\alpha(\mathrm{t}) \mathrm{S}(\mathrm{t}) \\
& b\left(S_{t}\right)=\sigma(t) S(t)
\end{aligned}
$$

La estructura del método numérico estocástico de Taylor 2.0 débil (Tocino, 2009) es:

$$
S_{n+1}=S_{n}+a \Delta+b \Delta W_{n}+\frac{1}{2} b b^{\prime}\left[\left(\Delta W_{n}\right)^{2}-\Delta\right]+b a^{\prime} \Delta Z+\frac{1}{2}\left[a a^{\prime}+\frac{1}{2} b^{2} a^{\prime \prime}\right] \Delta^{2}+\left(a b^{\prime}+\frac{1}{2} b^{2} b^{\prime \prime}\right)\{\Delta W \Delta-\Delta Z\}
$$


Donde, $\Delta=t_{n}-t_{n-1}=d t$, representa el tamaño del paso de cálculo y $\Delta W_{n}=W_{n}-W_{n-1}$ con $n=0,1,2, \ldots, N-1 ; S(0)=S_{0}$ representa el valor inicial o actual del activo subyacente. $\Delta Z$ representa la integral doble de ltô dada por,

$$
\Delta Z=I_{(1,0)}=\int_{\tau_{n}}^{\tau_{n+1}} \int_{\tau_{n}}^{s_{2}} d W_{s_{1}} d s_{2}
$$

En la ecuación (10), $\Delta Z$ está normalmente distribuida con media $E(\Delta Z)=0$, varianza $E\left((\Delta Z)^{2}\right)=(1 / 3) \Delta^{3} y$ covarianza $E(\Delta Z \Delta \mathrm{W})=(1 / 2) \Delta^{2}$. La covarianza entre las dos variables distribuidas normalmente $(\Delta \mathrm{W}, \Delta Z)$ se alcanza a través de dos variables aleatorias independientes $U_{1}$ y $U_{2}$ distribuidas $N(0,1)$ indicadas en (Kloeden y Platen, 1995; Tocino, 2009), mediante las transformaciones:

$$
\begin{aligned}
& \Delta W=U_{i} \sqrt{\Delta} \\
& \Delta Z=\frac{1}{2} \Delta^{3 / 2}\left(U_{1}+\frac{1}{\sqrt{3}} U_{2}\right)
\end{aligned}
$$

Se puede evitar la segunda variable aleatoria $\Delta Z$ en la ecuación (10) y utilizar sólo una única variable aleatoria $\Delta \widetilde{W}$ con propiedades de momento análogas a $\Delta W$ haciendo la sustitución,

$$
\Delta Z=\frac{1}{2} \Delta \widetilde{W} \Delta
$$

Resultando la aproximación débil de Taylor de orden 2.0 simplificada,

$$
S_{n+1}=S_{n}+a \Delta+b \Delta \widetilde{W}_{n}+\frac{1}{2} b b^{\prime}\left\{(\Delta \widetilde{W})^{2}-\Delta\right\}+\frac{1}{2}\left(a^{\prime} b+a b^{\prime}+\frac{1}{2} b^{\prime \prime} b^{2}\right) \Delta \widetilde{W}_{n} \Delta+\frac{1}{2}\left[a a^{\prime}+\frac{1}{2} a^{\prime \prime} b^{2}\right] \Delta^{2}
$$

Para aplicar el método de Taylor a la ecuación diferencial estocástica (7) sobre un intervalo [0,T], se debe discretizar el intervalo considerado, $\Delta=\mathrm{T} / \mathrm{N}$. Si en la ecuación diferencial estocástica $(7)$ se asume que $\alpha(\mathrm{t})=r$ y $\sigma(\mathrm{t})=\sigma$, siendo $\mathrm{r}$ y $\sigma$ constantes, donde $\mathrm{r}$ es igual a la tasa libre de riesgo y $\sigma$, es la volatilidad, se llega a la ecuación diferencial estocástica del precio del activo bajo el supuesto de riesgo neutral,

$$
d S(t)=r S(t) d t+\sigma S(t) d W(t)
$$

La ecuación (16) es considerada para valorar una opción estándar, por lo tanto, en términos del método numérico estocástico de Taylor 2.0 débil simplificado (Tocino, 2009), para la ecuación (16) se tiene que:

$$
S_{n+1}=S_{n}+r S_{n} \Delta+\sigma S_{n} \Delta \widetilde{W}_{n}+\frac{1}{2} S_{n} \sigma^{2}\left(\Delta \widetilde{W}_{n}^{2}-\Delta\right)+r \sigma S_{n} \Delta \widetilde{W} \Delta+\frac{1}{2}\left(r^{2} S_{n}\right) \Delta^{2}
$$

El método de Euler-Maruyama (Sauer, 2011) usa los tres primeros términos en la ecuación (17), esto es:

$$
S_{n+1}=S_{n}+r S_{n} \Delta+\sigma S_{n} \Delta \widetilde{W}_{n}
$$

Con la condición inicial $\mathrm{S}(0)=\mathrm{S}_{0}$.

\section{RESULTADOS Y DISCUSIÓN}

Se realizan diferentes experimentos numéricos usando la aproximación de Euler-Maruyama débil (EMD) y Taylor 2.0 débil (TAYD) dadas en las ecuaciones (17-18), comparando con el precio de la opción usando la fórmula de Black-Scholes representada en las ecuaciones (3-5), asumiendo en todos los experimentos numéricos el valor inicial del subyacente, $\mathrm{S}_{0}=42$. En las Tablas $1-2$ se presentan los resultados de diferentes simulaciones para determinar el valor de la prima, con volatilidades $\sigma=0.2, \sigma=0.3$ y $\sigma=0.4$, con diferentes precios de ejercicio $K=30,40$ y 50 . Se consideran diferentes pasos de tiempo de la forma $\mathrm{dt}=\mathrm{T} / \mathrm{N}=4 / 2^{\mathrm{p}}$ con $p=4,5, \ldots, 10$; siendo $T$ el tiempo de expiración y una tasa de interés libre de riesgo del $5 \%$. Los valores en media son presentados tomando diferentes caminos Brownianos $M=100,550$ y 1000 . Los resultados 
obtenidos con el método de referencia (Black y Scholes, 1973) dada por las ecuaciones (3-6) en la valoración de la opción considerando $\sigma=0.2$ y $K=30 ; \sigma=0.3$ y $K=40 ; \sigma=0.4$ y $K=50$ son de $U \$ 17.966 ; \cup \$ 14.159$ y U\$ 13.428 respectivamente. El método propuesto en este trabajo usando el esquema de Taylor con aproximación débil, los valores obtenidos en media son U\$18.554; U\$14.997 y U\$14.556, respectivamente.

Tabla 1: Valoración de la opción con el método Taylor débil 2.0 para $\sigma=0.2, \mathrm{~K}=30$

\begin{tabular}{|c|c|c|c|c|c|c|c|}
\hline $\mathrm{M}$ & $\mathrm{dt}=4 / 24$ & $\mathrm{dt}=4 / 25$ & $\mathrm{dt}=4 / 26$ & $\mathrm{dt}=4 / 27$ & $\mathrm{dt}=4 / 28$ & $\mathrm{dt}=4 / 29$ & $\mathrm{dt}=4 / 210$ \\
\hline 100 & 19.210 & 17.437 & 19.295 & 19.654 & 18.441 & 17.519 & 19.591 \\
\hline 550 & 18.634 & 18.621 & 18.102 & 18.668 & 18.627 & 18.591 & 18.861 \\
\hline 1000 & 18.523 & 18.146 & 18.605 & 18.274 & 18.375 & 18.136 & 18.318 \\
\hline
\end{tabular}

Tabla 2: Valoración de la opción con el método Taylor débil 2.0 para $\sigma=0.3, \mathrm{~K}=40$

\begin{tabular}{|c|c|c|c|c|c|c|c|}
\hline $\mathrm{M}$ & $\mathrm{dt}=4 / 2^{4}$ & $\mathrm{dt}=4 / 2^{5}$ & $\mathrm{dt}=4 / 2^{6}$ & $\mathrm{dt}=4 / 2^{7}$ & $\mathrm{dt}=4 / 2^{8}$ & $\mathrm{dt}=4 / 2^{9}$ & $\mathrm{dt}=4 / 2^{10}$ \\
\hline 100 & 16.764 & 14.013 & 16.883 & 17.059 & 15.355 & 12.472 & 16.081 \\
\hline 550 & 14.851 & 14.939 & 14.115 & 15.452 & 15.122 & 15.012 & 15.891 \\
\hline 1000 & 14.442 & 14.427 & 14.566 & 14.193 & 14.659 & 14.075 & 14.556 \\
\hline
\end{tabular}

Tabla 3: Valoración de la opción con el método Taylor débil 2.0 para $\sigma=0.4, \mathrm{~K}=50$

\begin{tabular}{|c|c|c|c|c|c|c|c|}
\hline $\mathrm{M}$ & $\mathrm{dt}=4 / 2^{4}$ & $\mathrm{dt}=4 / 2^{5}$ & $\mathrm{dt}=4 / 2^{6}$ & $\mathrm{dt}=4 / 2^{7}$ & $\mathrm{dt}=4 / 2^{8}$ & $\mathrm{dt}=4 / 2^{9}$ & $\mathrm{dt}=4 / 2^{10}$ \\
\hline 100 & 16.428 & 14.439 & 17.584 & 18.295 & 15.339 & 10.819 & 16.156 \\
\hline 550 & 13.779 & 14.298 & 13.150 & 15.208 & 14.606 & 14.471 & 16.131 \\
\hline 1000 & 13.074 & 13.815 & 13.878 & 12.893 & 14.193 & 13.270 & 13.841 \\
\hline
\end{tabular}

En la Tabla 4 se muestra el error cuadrático medio (ECM) en el cálculo del precio de la opción obtenida usando el método de Taylor débil 2.0, el método de Euler-Maruyama débil y el método de referencia usando la fórmula de $B S$ para una volatilidad $\sigma=0.2$, y diferentes grupos de trayectorias Brownianos $M=100,550$ y 1000. Los pasos de tiempo son considerados como en el experimento numérico anterior $\mathrm{dt}=\mathrm{T} / \mathrm{N}=4 / 2^{\mathrm{p}}$ con $p=4,5, \ldots, 10$ con diferentes precios de ejercicio $K$. Los errores cuadráticos medios son obtenidos comparando con el método de referencia dado por la fórmula de Black-Scholes. Los resultados confirman la precisión de los métodos propuestos usando el método de Euler-Maruyama débil simplificado y el esquema de Taylor de aproximación débil 2.0.

Tabla 4: Valor de la opción considerando el método propuesto con $\sigma=0.2$

\begin{tabular}{|c|c|c|c|c|}
\hline K & Valor Opción BS & M & ECM_EMD & ECM_TAYD \\
\hline \multirow{3}{*}{30} & \multirow{3}{*}{17.966} & 100 & 1.066 & 1.077 \\
\hline & & 550 & 0.561 & 0.567 \\
\hline & & 1000 & 0.277 & 0.301 \\
\hline \multirow{3}{*}{40} & \multirow{3}{*}{11.639} & 100 & 1.027 & 1.049 \\
\hline & & 550 & 0.571 & 0.580 \\
\hline & & 1000 & 0.177 & 0.177 \\
\hline \multirow{3}{*}{50} & \multirow{3}{*}{7.119} & 100 & 1.048 & 1.076 \\
\hline & & 550 & 0.496 & 0.505 \\
\hline & & 1000 & 0.142 & 0.127 \\
\hline
\end{tabular}


En la Tabla 5 se muestra el ECM usando los diferentes métodos para una volatilidad $\sigma=0.4$, el número de trayectorias Brownianos $M=100,550$ y 1000 . Los pasos de tiempo y precios de ejercicio $K$ son considerados como en el experimento numérico anterior. Los resultados muestran nuevamente la precisión de los métodos propuestos usando el método de Euler-Maruyama débil simplificado y el esquema de Taylor de aproximación débil 2.0. En particular, mejores aproximaciones se pueden obtener en los escenarios considerados cuando se usan volatilidades pequeñas con un mayor número de movimientos Brownianos.

Tabla 5: Valor de la opción considerando el método propuesto con $\sigma=0.4$

\begin{tabular}{|c|c|c|c|c|}
\hline $\mathrm{K}$ & Valor Opción BS & $\mathrm{M}$ & ECM_EMD & ECM_TAYD \\
\hline \multirow{3}{*}{30} & \multirow{3}{*}{21.128} & 100 & 2.870 & 2.909 \\
\cline { 3 - 5 } & & 550 & 1.553 & 1.564 \\
\hline \multirow{3}{*}{40} & \multirow{3}{*}{16.768} & 1000 & 0.474 & 0.459 \\
\cline { 3 - 5 } & & 100 & 2.903 & 2.950 \\
\cline { 3 - 5 } & & 550 & 1.464 & 1.474 \\
\hline \multirow{3}{*}{50} & \multirow{3}{*}{13.428} & 1000 & 0.521 & 0.490 \\
\cline { 3 - 5 } & & 100 & 2.840 & 2.890 \\
\cline { 3 - 5 } & & 550 & 1.387 & 1.396 \\
\cline { 3 - 5 } & & 1000 & 0.564 & 0.526 \\
\hline
\end{tabular}

En la Tabla 6 se presenta el valor de la opción para las tasas de interés libre de riesgo; $r=5 \%$ y $r=9 \%$, para diferentes grupos de trayectorias de movimientos Brownianos de tamaño $M=100,550$, 1000, para los diferentes pasos de tiempo $\mathrm{dt}=\mathrm{T} / \mathrm{N}=4 / 2^{\mathrm{p}}$ con $\mathrm{p}=4,5, \ldots, 10$. Considerando, el precio de ejercicio $K=30$ y la volatilidad $\sigma=0.4$. Los resultados indican que el valor de la prima aumenta con el aumento de la tasa de interés libre de riesgo como también aumenta los errores cuadráticos medios.

Tabla 6: Valor de la opción considerando el método propuesto $\operatorname{con} \sigma=0.4, K=30$

\begin{tabular}{|c|c|c|c|c|}
\hline$r(\%)$ & Valor Opción BS & $\mathrm{M}$ & ECM_EMD & ECM_TAYD \\
\hline \multirow{3}{*}{0.05} & \multirow{3}{*}{21.128} & 100 & 2.870 & 2.909 \\
\cline { 3 - 5 } & & 550 & 1.553 & 1.564 \\
\cline { 3 - 5 } & & 1000 & 0.474 & 0.459 \\
\hline \multirow{2}{*}{0.09} & \multirow{2}{*}{23.464} & 100 & 2.791 & 2.867 \\
\cline { 3 - 5 } & & 550 & 1.554 & 1.570 \\
\cline { 3 - 5 } & & 1000 & 0.554 & 0.508 \\
\hline
\end{tabular}

En la última simulación se considera la tasa libre de riesgo $r=5 \%$, el precio de ejercicio $K=30$, la volatilidad $\sigma=0.2$, el número de movimientos Brownianos $M=1000$. La Figura 1 presenta el precio de la opción para $T=2$, $\mathrm{dt}=2 / 1024$. La valoración de la opción usando el método de referencia es de $U \$ 15.107$, con el método de Euler-Maruyama de U\$15.370 con un error cuadrático medio de 0.264 , con la aproximación débil de Taylor 2.0 se obtiene un valor de la opción de U\$15.371 con error cuadrático medio de 0.264.

Para un tiempo de expiración $\mathrm{T}=4$ meses, $\mathrm{dt}=4 / 1024$, el valor de la opción con el método de referencia es U\$17.966, el método de Euler-Maruyama es U\$18.322 con un error cuadrático medio (ECM_EMD) de 0.355, con el método de Taylor es U\$18.318 con un error cuadrático medio (ECM_TAYD) de 0.357 como presentado en la Figura 2. Los resultados obtenidos con los diferentes experimentos numéricos permiten mostrar como el método propuesto usando el esquema de la aproximación débil de Euler-Maruyama y Taylor 2.0 son apropiados para valorar las opciones con un error cuadrático bajo, esto es con una buena aproximación. 


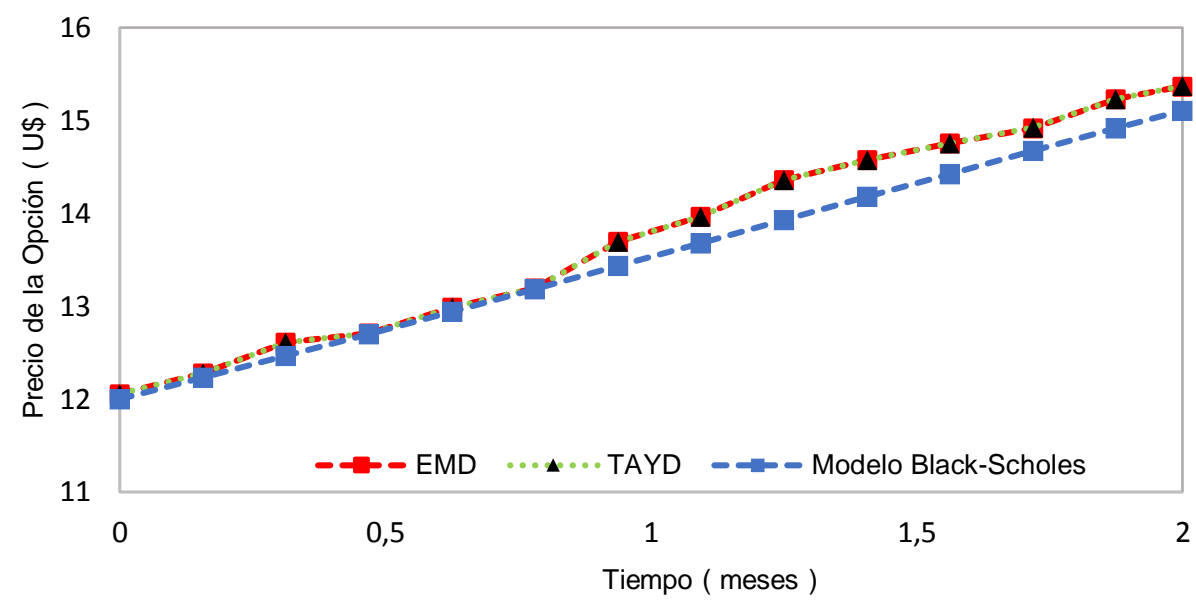

Fig. 1: Comparación del precio de la opción para T=2 meses

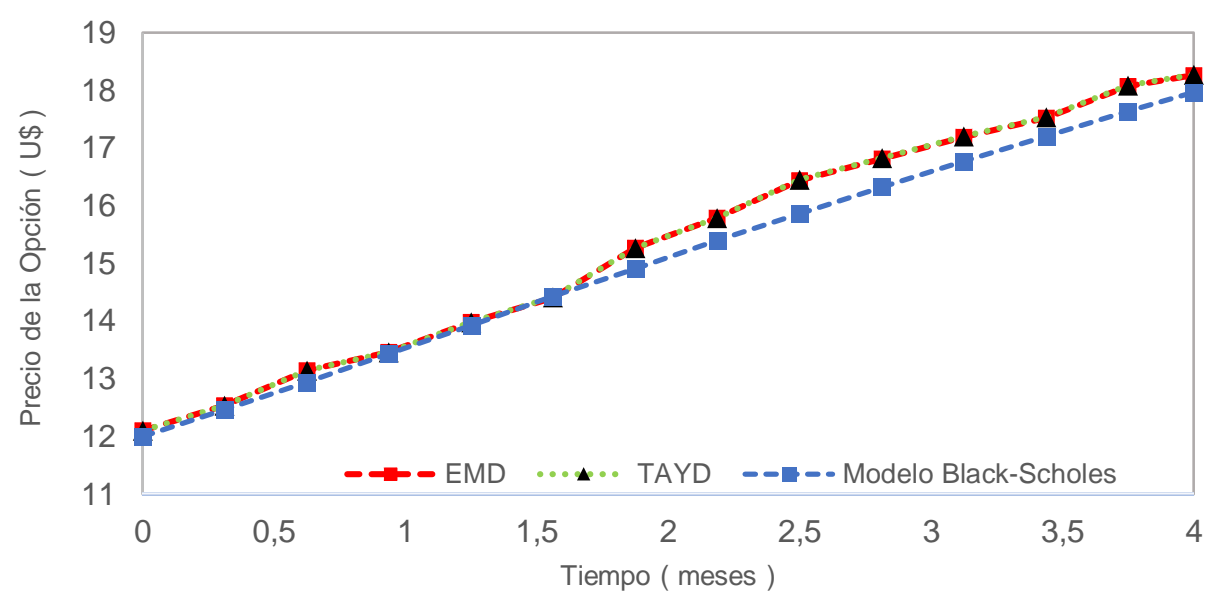

Fig. 2: Comparación del precio de la opción para T= 4 meses

\section{CONCLUSIONES}

Los métodos numéricos estocásticos débiles de Euler-Maruyama y Taylor de aproximación 2.0 fueron presentados para valoración de una opción de compra estándar con diferentes niveles de volatilidad, diferentes precios de ejercicio, con diferentes tasas de interés libre de riesgo y diferentes tiempos de expiración. Los métodos propuestos son validados con diferentes experimentos numéricos con varios pasos de tiempo y diferentes trayectorias Brownianas, el cálculo permite comparar el valor medio de la valoración de la opción de todas estas posibles trayectorias con relación al valor de la prima proporcionada por la fórmula de Black-Scholes. Los resultados muestran que los métodos débiles de Euler-Maruyama y Taylor de orden 2.0 presentan bajo error cuadrático medio en todos los escenarios analizados y, además, la precisión del valor de la prima es alta principalmente cuando el número de trayectorias aumenta, y el paso de tiempo es pequeño. El valor de la prima aumenta con el aumento de la tasa libre de riesgo, lo cual también origina que aumente el error cuadrático medio. El aumento en el tiempo de expiración de la opción también aumenta su valor. Finalmente, se muestra como los métodos de aproximación débiles son lo suficientemente precisos para usar en la ingeniería o en las finanzas.

\section{NOTACIÓN}

$\mathrm{S}_{0}=$ valor del activo subyacente al inicio del contrato

$\mu=$ tasa de retorno esperada

$\sigma=$ volatilidad del retorno del activo

$\mathrm{dW}$ = proceso de Wiener o movimiento Browniano

$\mathrm{K}=$ precio de ejercicio

$\mathrm{T}=$ tiempo hasta el vencimiento

$r=$ tasa de interés libre de riesgo
$\Delta=\mathrm{dt}=$ representa el tamaño del paso

ECM_EMD = error cuadrático medio con Euler-Maruyama

ECM_TAYD= error cuadrático medio con Taylor

$M=$ número diferentes de caminos Brownianos

$B S=$ Black y Scholes

$U \$=$ dólares americanos

$c=$ el precio de una opción europea de compra 


\section{REFERENCIAS}

Bermúdez, B., y Juárez, L., Solución Numérica de una Ecuación del Tipo Advección-Difusión, http://dx.doi.org/10.4067/S0718-07642014000100016, Información Tecnológica., 25(1), 151-160 (2014)

Black, F., y Scholes, M., The Pricing of Options and Corporate Liabilities, https://doi.org/10.1086/260062, J. Polit. Econ., 81(3), 637-654 (1973)

De la Cruz, M., Polupan, G., Martínez, E., y Carvajal, I., Estudio Numérico del Efecto de la Presión en el Proceso de Mezcla Metano-Oxígeno en un Arreglo de Chorros 4-Lug Bolt, http://dx.doi.org/10.4067/S0718-07642015000200018, Información Tecnológica., 26(2), 153-162 (2015)

De la Espriella, N.A., Casiano, G.R., y Ortega, C., Estudio Monte Carlo de un Ferrimagneto de Ising Mixto con Diferentes Anisotropías, http://dx.doi.org/10.4067/S0718-07642012000200014, Información Tecnológica., 23(2) 117-128 (2012)

Dos Santos, E.D., Xavier, C.M., y Petry, A.P., Estudio Numérico de Flujos Turbulentos Isotérmicos en Canales y Flujos Laminares con Convección Mixta en Cavidades, http://dx.doi.org/10.4067/S0718-07642011000100010, Información Tecnológica., 22(1), 71-82 (2011)

Huang, J., Zhu W., y Ruan, X., Option Pricing using the Fast Fourier Transform under the Double Exponential Jump Model with Stochastic Volatility and Stochastic Intensity, https://doi.org/10.1016/j.cam.2013.12.009, J. Comput. Appl. Math., 263, 152-159 (2014)

Kloeden, P. E., y Platen, E., Numerical Solution of Stochastic Differential Equations, Springer-Verlag, Heidelberg, Germany (1995)

Kwon, Y., y Lee, Y., A Second-Order Finite Difference Method for Option Pricing under Jump-Diffusion Models, https://doi.org/10.1137/090777529, SIAM J. Numer. Anal., 49(6), 2598-2617 (2011)

Li, S., Zhou, Y., Wu, Y., y Ge, X., Equilibrium Approach of Asset and Option Pricing under Lévy Process and Stochastic Volatility, https://doi.org/10.1177/0312896215619966, Aust. J. Manag., 42 (2), 276-295 (2016)

Mariani, M.C., SenGupta, I., y Bezdek, P., Numerical Solutions for Option Pricing Models Including Transaction Costs and Stochastic Volatility, https://doi.org/10.1007/s10440-012-9685-3, Act. Appl. Math., 118(1), 203-220 (2012)

Milstein, G.N., Weak Approximation of Solutions of Systems of Stochastic Differential Equations, Theory Probab. Appl., 30, 750-766 (1985)

Monoyios, M., Option Pricing with Transaction Costs using a Markov Chain Approximation, https://doi.org/10.1016/S0165-1889(03)00059-9, J. Econ. Dyn. Cont., 28 (5), 889-913 (2004)

Nowak, P., y Romaniuk, M., Application of Levy Processes and Esscher Transformed Martingale Measures for Option Pricing in fuzzy framework, https://doi.org/10.1016/j.cam.2013.11.031, J. Comput. Appl. Math., 263, 129-151 (2014)

O'Sullivan, S., y O'Sullivan, C., On the Acceleration of Explicit Finite Difference Methods for Option Pricing, https://doi.org/10.1080/14697680903055570, Quant. Finan., 11 (8), 1177-1191 (2011)

Ortega, A.J., Pires, L.F.G., y Nieckele, A.O., Una Alternativa para la Simulación Numérica del Comportamiento Térmico en Régimen Transitorio de Flujo de Gas en Redes de Ductos, http://dx.doi.org/10.4067/S0718-07642009000500011, Información Tecnológica., 20(5), 83-88 (2009)

Palma, J.A., Barbosa, J.M.A., y Silva, A.A., Simulación Numérica de la Propagación de una Fisura en un Material Degradado por Efecto de la Fragilización por Hidrógeno, http://dx.doi.org/10.4067/S0718-07642010000200009, Información Tecnológica., 21(2), 65-76 (2010)

Rana, U., y Ahmad, A., Numerical Solution of Pricing of European Put Option with Stochastic Volatility, Int. J. Eng., 24 (4), 189-202 (2011)

Sauer, T., Numerical Solution of Stochastic Differential Equations in Finance, https://doi.org/10.1007/978-3-642-172540_19, Hand. Comput. Statistic. 529-550 (2011)

Shi, G., Liu, X., y Tang, P., Pricing Options under the Non-Affine Stochastic Volatility Models: An Extension of the HighOrder Compact Numerical Scheme, https://doi.org/10.1016/j.frl.2015.12.004, Finan. Res. Lett., 16, $220-229$ (2016)

Suescún-Díaz, D., Oviedo-Torres, Y.M., y Girón-Cruz, L.E., Solution of the Stochastic Point Kinetics Equations Using the Implicit Euler-Maruyama Method, https://doi.org/10.1016/j.anucene.2018.03.013, Ann. Nucl. Energy., 117, 45-52 (2018)

Tocino, A., Simplified Order 4.0 Weak Taylor Schemes for Additive Noise, https://doi.org/10.1016/j.cam.2009.02.002, J. Comput. Appl. Math., 231(1), 154-159 (2009)

Toivanen, J., Numerical Valuation of European and American Options under Kou's Jump-Diffusion Model, https://doi.org/10.1137/060674697, SIAM J. Scientific Comput., 30 (4), 1949-1970 (2008)

Zhang, S., y Wang, L., Fast Fourier Transform Option Pricing with Stochastic Interest Rate, Stochastic Volatility and Double Jumps, https://doi.org/10.1016/j.amc.2013.05.008, Appl. Math. Comput., 219(23), 10928-10933 (2013)

Zhu, S.P., Badran, A., y Lu, X., A New Exact Solution for Pricing European Options in a Two-State Regime-Switching Economy, https://doi.org/10.1016/j.camwa.2012.08.005, Comput. Math. Appl., 64(8), 2744-2755 (2012) 